\title{
Nasal Ulceration Reveling Multifunctional Tuberculosis
}

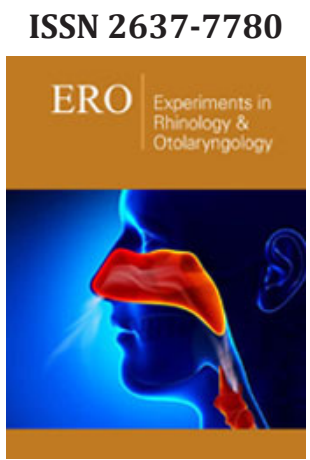

*Corresponding author: Fatogoma Issa Koné, Department of ENT-Head and Neck Surgery, University hospital of Gabriel Touré, Mali

Submission: 眥 July 23, 2020

Published: 俔August 10, 2020

Volume 3 - Issue 2

How to cite this article: Djibril Samaké, Fatogoma Issa Koné, et al. Nasal Ulceration Reveling Multifunctional Tuberculosis. Exp Rhinol Otolaryngol 3(2). ERO.000558.2020.

DOI: 10.31031/ERO.2020.03.000558

Copyright@ Fatogoma Issa Koné, This article is distributed under the terms of the Creative Commons Attribution 4.0 International License, which permits unrestricted use and redistribution provided that the original author and source are credited.

\author{
Djibril Samaké ${ }^{1}$ Fatogoma Issa Koné2*, Naouma Cissé ${ }^{2}$, Djeneba Konaté ${ }^{3}$, Kolo \\ Diamouténé $^{4}$, Kadidiatou Singaré ${ }^{2}$, Samba Karim Timbo ${ }^{2}$ and Keïta Mohamed \\ Amadou $^{2}$ \\ ${ }^{1}$ Reference Health Center Town V, ENT-Head and Neck surgeon, Mali \\ ${ }^{2}$ Department of ENT-Head and Neck Surgery, University hospital of Gabriel Touré, Mali \\ ${ }^{3}$ Department of pediatrics, University hospital of Gabriel Touré, Mali \\ ${ }^{4}$ ENT-Head and Neck surgeon, Regional hospital Gao, Mali
}

\begin{abstract}
Objectives: We wanted to discuss the aetiopathogenic, diagnostic and therapeutic of nasal tuberculosis aspect.

Clinical case: This is a 10-year-old patient admitted for nasal blistering ulceration. The diagnosis of nasal tuberculosis was retained after histopathological examination. After the clinical and paraclinical investigations, we retained multifocal tuberculosis in front of tuberculosis of the ear, pulmonary and a pain of pott. The therapeutic antituberculous inclusion to the two-month regimen of rifampicin + isoniazid + pyrazinamide + ethambutol and 4-month rifampicin + isoniazid. We noted a clear improvement at four months of treatment, nasal, pulmonary, otological lesions but with sequelae at the dorsal level. Any recurrence after one-year decline.
\end{abstract}

Conclusion: Nasal tuberculosis, although rare, remains a reality in our country. In front of a nasal ulceration and a nasal obstruction must make search for nasal tuberculosis. Careful clinical examination can detect more complications.

Keywords: Nasal ulcer; Multifocal tuberculosis; Histology

\section{Introduction}

Tuberculosis (TB) remains a public health problem [1]. It is one of the oldest diseases of humanity and a source of morbidity and mortality [2]. There are nearly 9 million new cases and 2 million TB deaths worldwide each year [3]. Extra-pulmonary tuberculosis accounts for $10 \%$ of adult TB, depending on race, ethnic group, age, immune status [4]. The extrapulmonary TB in seropositive patients (human immunodeficiency virus) accounts for up to 53 to 62 percent of TB cases [2]. Tuberculosis can affect all organs of the body, except the nails, hair, teeth [2]. Tuberculosis of the head and neck usually affects the lymph nodes, pharynx, and larynx [5]. Tuberculosis of the head and neck represents $10 \%$ of the causes of extra-pulmonary tuberculosis [6]. Tuberculosis of the nose, nasopharynx, and nasosinus cavities is rare. Giovanni Morgagni described nasal tuberculosis in 1761 for the first time on an autopsy of a patient with ulceration of the nose, palate and nasopharynx that had concomitant pulmonary affections [1]. Antoni Bruzgielewicz reported that tuberculosis of the nose and sinuses, accounted for 4, 1\% among 73 cases of head and neck tuberculosis [7]. The multifocality of tuberculosis remains a little discussed entity especially in the head and neck region. Tuberculosis of the head and neck raises many interests and many fields of research:

A. Insidious and polymorphic clinical manifestations.

B. Multifocal involvement of the organs of the head and neck.

C. The similarity with the ENT cancerous lesions and the diagnostic error are sources of diagnostic delay. 
The association of nasal tuberculosis, sore throat, pulmonary tuberculosis and the ear on immunocompetent ground is rare. We report a rare case of multifocal tuberculosis localization by discussing the clinical, diagnostic, and therapeutic aspects in our context.

\section{Case Report}
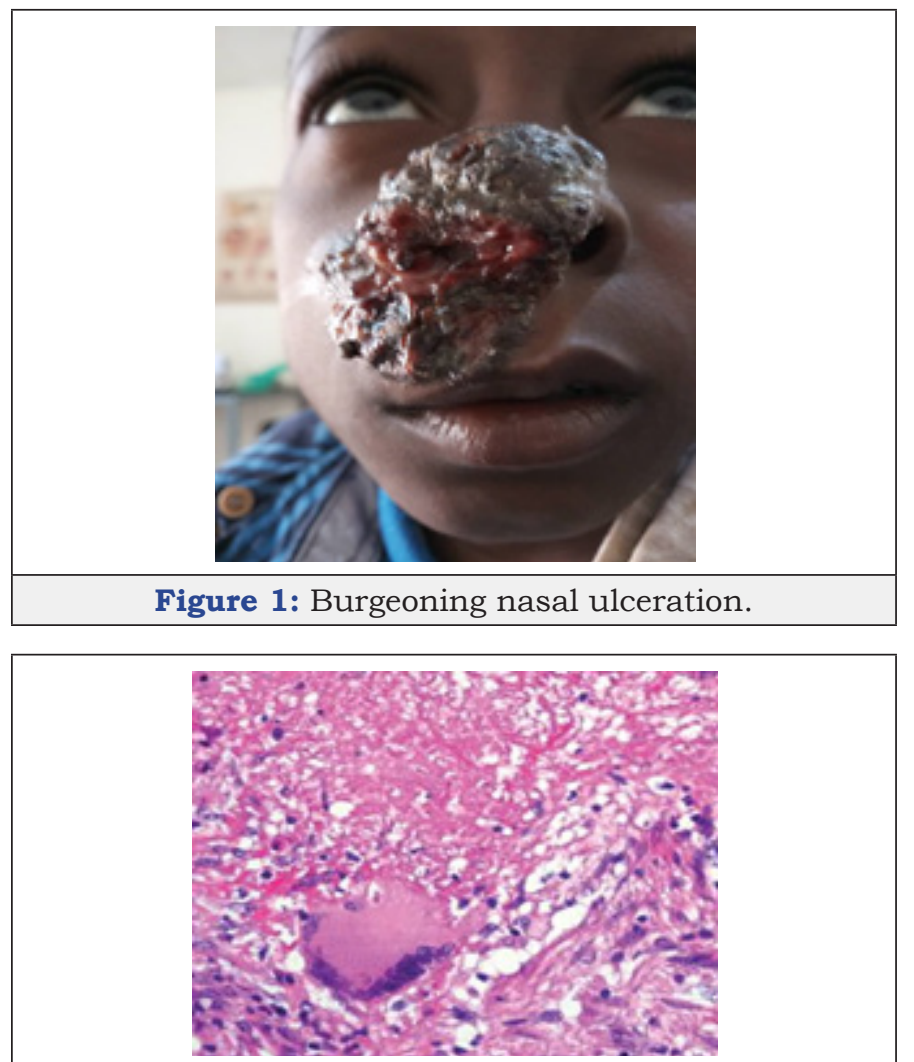

Figure 2: (HE x 400) Tuberculosis showing lymphocytes, epithelioid cells, giant Langhans cells and caseous necrosis.

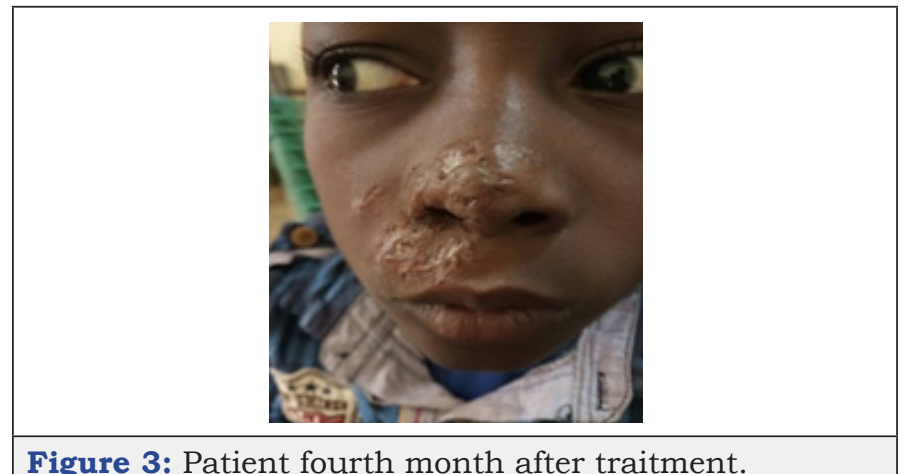

This is a 10 years old patient admitted for nasal ulceration. The clinical history dates back three months to the insidious installation of bilateral nasal obstruction associated with fetid purulent rhinorrhea. In these antecedents no notion of tuberculous contagion was noted, a BCG vaccination scar was found on the left arm. The HIV status was negative, and we did not note any family history of nasal tuberculosis. At admission, the patient had a good general condition. The rhinological examination noted a budding ulcerous swelling (Figure 1) of the nasal pyramid. Otoscopy revealed polyps filling the two external auditory canals. The remainder of the clinical examination found a dorsal kyphoscoliosis. At the end of this physical examination, we performed a thoracoabdominal computed tomography that objectified a Pott's disease. Chest X-ray revealed poorly heterogeneous opacities of the left lung associated with ipsilateral pleuritis. The blood count showed a hypochromic microcytic anemia with thrombocytosis. Tuberculin intradermal reaction was phlyctenular at $15 \mathrm{~mm}$ and anatomopathology of the cutaneous biopsy of the nasal pyramid revealed gigantocellular follicles with caseous necrosis indicating tuberculosis (Figure 2). We concluded that nasal tuberculosis was multifocal tuberculosis. The patient received a six-month antituberculous treatment with the two months regimen of Rifampicin + Isoniazid + Pyrazinamide + Ethambutol and four months Rifampicin + Isoniazid. We noted a net regression of the lesions at four months after treatment (Figure 3). Any recurrence after one year.

\section{Discussion}

Tuberculosis is a public health problem and its incidence is decreasing with the advent of antituberculous chemotherapy. Tuberculosis can be localized on any part of the body except uncles, hair and teeth [2]. It occurs following the direct inoculation of a nose prick, tuberculosis germ or haematogenous spread of a primary infection [5]. Nasal tuberculosis can be primary or secondary, following pulmonary tuberculosis [8]. Its nasal location is rare [5]. This rarity is explained by the ciliary movement, the bactericidal effect of the secretions by filtering by nasal vibrissae and the inherent resistance offered by the nasal mucosa [1]. In our case tuberculosis was secondary and integrated into multifocal tuberculosis involving nasal, pulmonary, dorsal and middle ear tuberculosis. Multifocality is rarely reported in the literature and remains a little discussed entity. In the Monga [3] series of 48 tuberculosis cases, three cases of nasosinus tuberculosis were identified including three cases of multifocal tuberculosis [3]. The involvement of the middle ear in our case is not the exception although rare. It has an incidence of $0.05 \%$, in the series of Soumyajit D [2]. In $52.9 \%$ the tuberculosis sitting at the level of the ENT sphere was primary in the series of Rithika [6]. The patient presented a long consultation time, this long consultation time is the prerogative of nasal tuberculosis. Similarity to other ENT diseases is a source of diagnostic error. The consultation period varied from eight to 47 months in the Pai Pang series with an average delay of 4 months [9]. The clinical spectrum has been variable in our case. The absence of fever and the alteration of the general condition in our case is not the exclusive property of tuberculosis. In several series fever and weight are the common signs whatever the location. Rithika found fever and swelling as a primum sign [6]. Based on the review of the literature, we noted variability in the clinical spectrum and this variability is a function of the location of TB.

In our patient, the spectrum presented a budding ulceration of the nasal pyramid with filling of the two nasal cavities and rhinorrhea, which corresponded to the ulcerative form of nasal tuberculosis. It can manifest as a nasal obstruction, epistaxis, and purulent or bloody, and when the paranasal sinus is involved, the evolution can be marked by bone destruction with corollary the 
appearance of ophthalmological manifestations such as diplopia and exophthalmia [9]. It can also be in the form of a recurrent polyp [8].

\section{Nasal tuberculosis has three forms [5]:}

a. Lupus vulgaris, or nodular form, is in the form of papules and scabs on the nasal vestibule and adjacent skin.

b. The ulcerous form involves the cartilaginous nasal septum or inferior turbinate and presents nasal obstruction, crust, flow, epistaxis.

c. Sinus granuloma, which presents itself as a mass in the paranasal sinuses with osteomyelic changes [5].

The diagnosis of tuberculosis was based in our case on histopathological examination after biopsy revealing a gigantocellular granuloma with caseous necrosis. It allowed making the diagnosis. The orientative elements have been reported it is the tuberculin intradermal reaction which was positive at $15 \mathrm{~mm}$. A definitive diagnosis is made by isolating tubercle bacilli from tissue samples obtained by biopsy or surgery [1]. This diagnosis can be carried out as in our case on the histological aspect highlighting a Langhans giant cell epithelioid granuloma [5].

The multifocality of tuberculosis in our case was an important morbidity and was a source of diagnostic wandering. Multiple pathologies come into confrontation with tuberculosis. Nose conditions such as rhinoscleroma, Wegener granulomatosis should be investigated. The histology allows establishing the diagnosis by highlighting Mikulicz cells for rhinoscleroma. Computed tomographic investigations can give an orientation towards the other affections. Imaging characteristics, destruction, invasion and necrosis point to malignant lesions, granulomatous reactions, metastases, fungal infections [1]. Therapeutic inclusion was established on the combination of Rifampicin + Isoniazid + Pyrazinamide + Ethambutol (RHZE) at 2 months and Rifampicin + Isoniazid (RH) at 4 months of treatment. Our six-month regimen joins that of Poonam S [5]. We adopted the same pattern as Seyed Mohammad Alavi on the basis of two months of RHEZ and four months of HR [8]. The post-therapeutic evolution was simple with regression of the nasal, otological, pulmonary lesions, and with persistence of dorsal sequelae. The post-therapeutic consequences have been simple for many authors $[1,8]$. The treatment of nasal tuberculosis in our case was integrated in the context of multifocal tuberculosis.

\section{Conclusion}

Nasal tuberculosis, although rare, remains a reality in our country. It can integrate in a multifocal setting; it can be primary or secondary. In front of a nasal ulceration and a nasal obstruction must make search for nasal tuberculosis. It is important to note that a clinical examination and a well-researched semiology make it possible to detect other localizations. The histology makes it possible to highlight a gigantocellular cell with caseous necrosis. Diagnosis and early management help prevent complications.

\section{Conflict of Interest}

The authors did not declare any conflict interest.

\section{References}

1. Darshan KB, Ajay KV, Riddhi J, Surya K, Anand P, et al. (2016) Tip of nose tuberculosis: A rare presentation of extra pulmonary tuberculosis. Intractable \& Rare Diseases Research 5(2): 133-136.

2. Soumyajit D, Debajit D, Uttal T, Bhuyan NS (2016) Head and neck tuberculosis: Scenario in a tertiary care hospital of north eastern India. Journal of Clinical and Diagnostic Research 10(1): 04-07.

3. Monga S, Malik JN, Jan S, Bahadur B, Jetley S, et al. (2017) Clinical study of extrapulmonary head and neck tuberculosis in an urban setting. Acta Otorhinolaryngologica Italica 37: 493-499.

4. Robert L, Marc L, Alimuddin Z (2015) Clinical aspects of adult tuberculosis. Cold Spring Harb Perspect Med 6: 1-25.

5. Poonam S, Vikram W, Ishwar S, Varuna M, Pragya R (2018) Naso-septal tuberculosis mimicking dermoid in a 3-year old child. Iranian Journal of Otorhinolaryngology 30(4): 231-235.

6. Rithika S, Kiran MB (2017) Manifestations of tuberculosis in otorhinolaryngology practice: A retrospective study conducted in a coastal city of south India. Indian J Otolaryngol Head Neck Surg 69(2): 210-215.

7. Antoni B, Anna R, Ewa Osuch-Wójcikewicz, Kazimierz N, Rafał C (2014) Tuberculosis of the head and neck-epidemiological and clinical presentation. Arch Med Sci 10(6): 1160-1166.

8. Seyed MA, Roohangiz N (2014) Nasal tuberculosis in a 56-year-old woman. Caspian J Intern Med 5(1): 49-51.

9. Pai P, Weiyi D, Shuchun L, Shuang B, Yanan M, et al. (2018) Clinical study of tuberculosis in the head and neck region-11 years' experience and a review of the literature. Emerging Microbes \& Infections 7(4): 1-10. 